\title{
Identification of the Leishmania major Proteins LmjF07.0430, LmjF07.0440, and LmjF27.2440 as Components of Fatty Acid Synthase II
}

\begin{abstract}
Aner Gurvitz
Section of Physiology of Lipid Metabolism, Center for Physiology, Pathophysiology and Immunology, Institute of Physiology, Medical University of Vienna, Schwarzspanierstrasse 17, 1090 Vienna, Austria

Correspondence should be addressed to Aner Gurvitz, aner.gurvitz@meduniwien.ac.at

Received 15 July 2009; Accepted 23 October 2009

Recommended by Wuyuan Lu

Leishmania major causes leishmaniasis and is grouped within the Trypanosomatidae family, which also includes the etiologic agent for African sleeping sickness, Trypanosoma brucei. Previous studies on T. brucei showed that acyl carrier protein (ACP) of mitochondrial fatty acid synthase type 2 (FASII) plays a crucial role in parasite survival. Additionally, 3-oxoacyl-ACP synthase TbKASIII as well as TbHTD2 representing 3-hydroxyacyl-ACP dehydratase were also identified; however, 3-oxoacyl-ACP reductase TbKAR1 has hitherto evaded positive identification. Here, potential Leishmania FASII components LmjF07.0440 and LmjF07.0430 were revealed as 3-hydroxyacyl-ACP dehydratases LmHTD2-1 and LmHTD2-2, respectively, whereas LmjF27.2440 was identified as LmKAR1. These Leishmania proteins were ectopically expressed in Saccharomyces cerevisiae htd2 $\Delta$ or oar1 $\Delta$ respiratory deficient cells lacking the corresponding mitochondrial FASII enzymes Htd2p and Oarlp. Yeast mutants producing mitochondrially targeted versions of the parasite proteins resembled the self-complemented cells for respiratory growth. This is the first identification of a FASII-like 3-oxoacyl-ACP reductase from a kinetoplastid parasite.
\end{abstract}

Copyright (c) 2009 Aner Gurvitz. This is an open access article distributed under the Creative Commons Attribution License, which permits unrestricted use, distribution, and reproduction in any medium, provided the original work is properly cited.

\section{Introduction}

Leishmania species represent a group of parasitic protozoa that cause widespread morbidity and even mortality (http://www.who.int/tdr/diseases/leish/diseaseinfo.htm), which are transmitted to humans through the bites of sandflies (for reviews on visceral, cutaneous, and mucosal leishmaniases, see $[1,2])$. Leishmania spp. are clustered within the Trypanosomatidae family that additionally contains Trypanosoma spp. and Crithidia fasciculata. The World Health Organisation estimates that approximately 12 million people suffer from various forms of leishmaniasis, some of which are lethal if left untreated. It is estimated that about 350 million people are at risk worldwide, and human infections are found in 16 countries in Europe (http://www.who.int/tdrold/diseases/leish/files/leish-poster. pdf). As a genus, Leishmania represents a major global human disease agent. Apparently, there is a rise in the rate of new Leishmania infections, and Leishmania/HIV coinfection has been termed as an emerging disease in southern Europe.
Moreover, drug resistance has also been reported recently. Since most available drugs are expensive, and treatment is long and accompanied by severe side effects but at the same time becoming less effective, there is a renewed interest and urgency in developing new antileishmanial drugs.

An attractive target process for novel drug design is represented by the type 2 fatty acid synthase (FASII) system $[3,4]$, which occurs in the mitochondria of trypanosomatids [5]. Despite the fact that the L. major genome was completely sequenced in 2005 [6], there is currently no information available on mitochondrial FASII in this particular kinetoplastid parasite. Pioneering studies on $T$. brucei revealed that it has an essential mitochondrial FASII process, which it employs for the production of octanoate and palmitate [7]. It was found that RNAi depletion of the mitochondrial acyl carrier protein (ACP) significantly reduced cytochrome-mediated respiration in the procyclic form of trypanosomes, and effected changes in mitochondrial morphology and alterations in the membrane potential $[7,8]$. In addition to ACP, the T. brucei FASII enzymes 
TABLe 1: Plasmids and oligonucleotides used.

\begin{tabular}{|c|c|c|}
\hline Plasmids or oligonucleotides & Description & Sources or references \\
\hline \multicolumn{3}{|l|}{ plasmid $^{\mathrm{a}}$} \\
\hline 1] pBluescript II KS & pKS cloning vector & Stratagene \\
\hline pKS:LmjF07.0440 & LmjF07.0440 in pBluescript (with primers for $L m H T D 2-1$ ) & This study \\
\hline pKS:LmjF07.04301 & LmjF07.0430 in pBluescript (with primers for LmHTD2-2) & This study \\
\hline pKS:LmjF27.2440 & LmjF27.2440 in pBluescript (with primers for LmOAR1-1) & This study \\
\hline pKS:LmjF24.2030 & LmjF24.2030 in pBluescript (with primers for $L m$ OAR1-2) & This study \\
\hline 2] YEp352 & URA3-marked multicopy plasmid & {$[17]$} \\
\hline 3] YEp:CTA1 ${ }^{2}$ & CTA1 behind its promoter (pYE352:CTA1) & {$[18]$} \\
\hline YEp:LmjF07.04403 & LmjF07.0440 behind the CTA1 promoter & This study \\
\hline YEp:LmjF07.0430 3 & LmjF07.0430 behind the CTA1 promoter & This study \\
\hline YEp:LmjF27.2440 & LmjF27.2440 behind the CTA1 promoter & This study \\
\hline YEp:LmjF24.2030³ & LmjF24.2030 behind the CTA1 promoter & This study \\
\hline YEp:OAR $1^{3}$ & OAR1 behind the CTA1 promoter & {$[14]$} \\
\hline YEp:YHR067w3 & HTD2 behind the CTA1 promoter & {$[12]$} \\
\hline \multicolumn{3}{|l|}{ oligonucleotide ${ }^{\mathrm{b}}$} \\
\hline Lm HTD2-1 5' NcoI & TTATCCATGGAAGCGGCAGCACGCCGCATCATC & This study \\
\hline Lm HTD2-1 3' HindIII & TATTAAGCTTAAAGCCGACGCTCCACTCGCTC & This study \\
\hline Lm HTD2-2 5' NcoI & TTATCCATGGCCAGCGAGACTGCACCACTTGTC & This study \\
\hline Lm HTD2-2 3' HindIII & TATTAAGCTTACTTCGTCACAGACCACTCACTC & This study \\
\hline Lm OAR1-1 5' NcoI & TTATCCATGGGTGCCCСTAACGCACACCСAC & This study \\
\hline Lm OAR1-1 3' HindIII & TATTAAGCTTACGATAAGCCGCCATCGACGGCG & This study \\
\hline Lm OAR1-2 5' NcoI & TTATCCATGGACGCCAAGTCCATCCAGTCG & This study \\
\hline Lm OAR1-2 3' HindIII & TATTAAGCTTACTTGGCTGCCATACGGGTTGCC & This study \\
\hline
\end{tabular}

${ }^{a}$ The numbers in superscript following the plasmids' designation refer to their parental vectors, for example, pKS:LmjF07.0440 ${ }^{1}$ was derived from 1] pBluescript II KS.

${ }^{b}$ Lm HTD2-1 refers to LmjF07.0440, Lm HTD2-2 to LmjF07.0430, Lm OAR1-1 to LmjF27.2440, and Lm OAR1-2 to LmjF24.2030.

3-oxoacyl-ACP synthase KASIII [7] and 3-hydroxyacylACP dehydratase HTD2 [9] have also been identified. Furthermore, mitochondrial compartmentalisation of three FabG candidates of T. brucei FASII, annotated as KAR1, KAR2, and KAR3, has been convincingly demonstrated using fusions with green fluorescent protein GFP [7]; however, their physiological function as 3-oxoacyl-ACP reductases of mitochondrial FASII has hitherto not been reported. Thus, it is important to identify Leishmania KAR1 so as to complete our picture of mitochondrial FASII in trypanosomatids, and in doing so to gain a better understanding of the enzymes undertaking this process, thereby refining our appreciation of their potential suitability for novel drug design.

A convenient and safe method for revealing the identity of parasite proteins is to demonstrate their physiological function in Saccharomyces cerevisiae mutant cells missing the corresponding yeast enzymes. In fungi, FASII is a mitochondrial process that is critical for organellar function and morphology, and a lesion in any one of the genes encoding FASII proteins results in a respiratory growth phenotype, underdeveloped mitochondria lacking cytochromes, and, in some cases, loss of mitochondrial DNA (reviewed in $[10,11])$. For instance, yeast $h t d 2 \Delta$ mutants devoid of the mitochondrial FASII enzyme 3-hydroxyacyl-ACP dehydratase Htd2p [12] were used previously to identify T. brucei
HTD2 [9]. Another more recent example of how yeast FASII mutants were employed as a surrogate host for functional complementation studies is represented by the manner in which oar1s mutant cells lacking the mitochondrial 3oxoacyl-ACP reductase Oarlp [13] had helped to identify the human and nematode counterpart reductases $[14,15]$.

Here, S. cerevisiae was exploited further as a heterologous system for determining whether candidate $L$. major HTD2 and KAR1 proteins might represent physiologically functional FASII enzymes. This was done by monitoring the potential interchangeability of ectopically expressed Leishmania proteins that were preceded by a mitochondrial leader sequence (MLS) with the analogous fungal enzymes Htd2p or Oarlp in the aforementioned respiratory deficient strains ( $h t d 2 \Delta$ and oar $1 \Delta$ mutant cells) during growth on glycerol medium. The results are viewed from the perspective of the utility of yeast FASII mutants for identifying Leishmania FASII enzymes.

\section{Materials and Methods}

2.1. S. cerevisiae Strains, Plasmids, and Oligonucleotides. Plasmids and oligonucleotides used are catalogued in Table 1. The Escherichia coli strain TOP10 F' (Invitrogen, Carlsbad, CA, USA) was exploited for all plasmid multiplications 


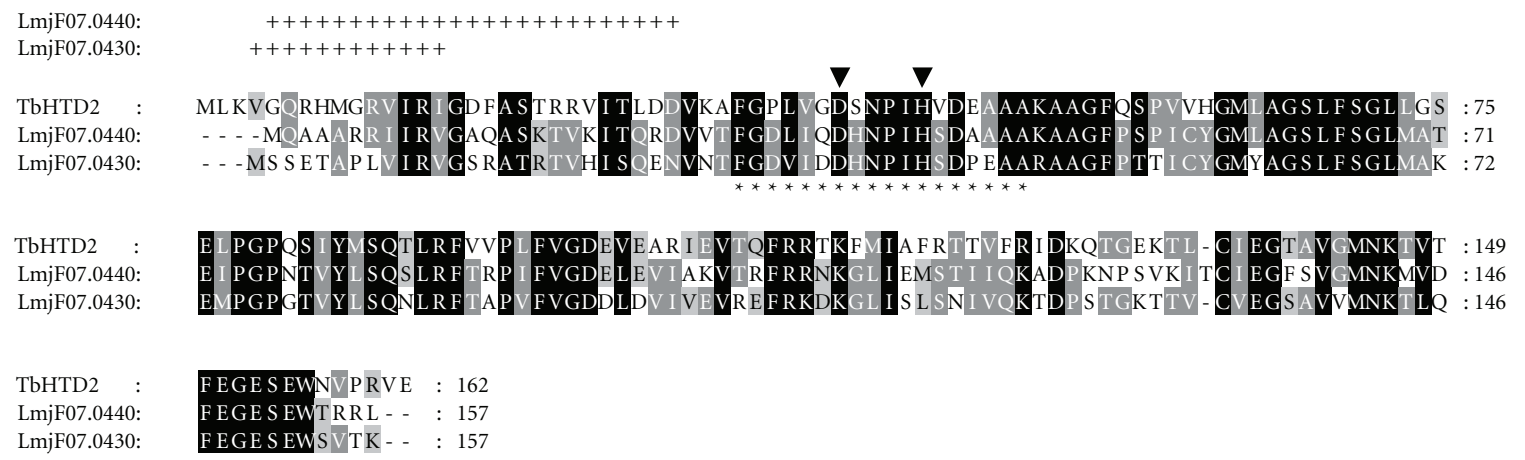

FIgure 1: Similarity of L. major LmjF07.0440 and LmjF07.0430 to their T. brucei homologue, TbHTD2. Explanations for this and Figure 3 are given. Multalin- and Genedoc-based comparison of the deduced amino acid sequences is provided. Dashes indicate the arrangement of the sequences for best fit. Black shadings refer to conserved amino acid residues among all three sequences whereas the darker and lighter grey shadings denote regions with more relaxed residue similarities not necessarily shared by the full set of sequences. MitoProt predictions of cleaved mitochondrial leader sequences are indicated (+). Active site residues of human mitochondrial dehydratase D62 and H67 are denoted $(\boldsymbol{\nabla})$, whereas the hydratase 2 domain is underscored $(*)$. The sequences used for the Leishmania proteins throughout this work were retrieved from GeneDB of the Sanger Institute, whereas the accession number for TbHTD2 was Q580H9_9TRYP.

and purifications. The mutant yeast strains BY4741oar1 $\Delta$

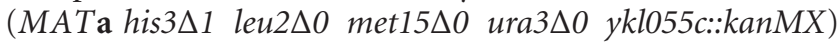
and BY4741htd2 $\Delta$ (yhr067w::kanMX) were acquired from EUROSCARF (http://www.uni-frankfurt.de/). Expression plasmids were incorporated into yeast cells using a reported procedure [16], and transformants were kept on SD-Ura medium, outlined below.

2.2. Plasmid Constructions. DNA inserts were assembled within plasmids according to standard methods [19]. The LmjF07.0430, LmjF07.0440, LmjF27.2440, and LmjF24.2030 genes were amplified from genomic $L$. major DNA by polymerase chain reaction (PCR) and the corresponding oligonucleotide pairs (Table 1). Sequential electrophoresis, processing, and tethering of amplicons to an EcoRV-digested pBluescript II plasmid vector (Stratagene, La Jolla, CA) are described [20]. The inserts were purified following digestion of the recombinant pBluescript plasmids with NcoI and HindIII restriction enzymes and fitted within a previously published plasmid YEp352:CTA1 [18] that is based on a URA3-marked YEp352 multicopy vector [17]. These inserts were placed behind the CTA1 promoter as gene fusions with the nucleotides for a cleavable N-terminal yeast MLS directing Coq3p to the organelle [21], as described in [20]. Nucleotide sequencing of the pBluescript inserts ensured that no mutations were introduced during the PCR amplification step. In addition, this procedure was also applied to the YEp352-based expression plasmids, verifying that the reading frame across the fusion boundary between the L. major genes and the yeast COQ3 nucleotides encoding the MLS was intact. Plasmid YEp352:OAR1 expressing yeast OAR1 as well as plasmid YEp352:YHR067w producing fungal Hdt2p are both described $[12,14]$.

2.3. Media, Enzyme Assays, Respiration Competence, and Lipoic Acid Measurements. Standard media were made for yeast [22] and E. coli [23]. URA3-marked multicopy plasmids were kept in transformed S. cerevisiae strains by bearing upon the cells selective pressure using solid SD-Ura medium. This medium consisted of $0.67 \%$ (wt/vol) yeast nitrogen base without amino acids, 2\% (wt/vol) D-glucose, 3\% (wt/vol) agar, and with all necessary supplements added with the exception of uracil (Sigma-Aldrich Inc., St. Louis, MO, USA). Solid synthetic complete glycerol medium (SCglycerol) was processed essentially as the previous SD-Ura medium, with the only two differences being the addition of uracil and replacement of glucose with 3\% (wt/vol) glycerol as the exclusive carbon source. Respiration competence was tested using an overlay made with $0.1 \%$ (wt/vol) 2,3,5triphenyltetrazolium chloride, (TTC) [24]. Lipoic acid was determined using a biological method that followed the growth of a lipoic acid-deficient E. coli strain in response to the presence of lipoic acid in yeast cells, with each yeast strain being propagated in triplicate cultures $[25,26]$.

2.4. Mitochondrial Localisation Predictions and Sequence Comparisons. MitoProt (http://ihg2.helmholtz-muenchen. de/ihg/mitoprot.html), PSORT II (http://psort.ims.utokyo.ac.jp/form2.html), and TargetP (http://cbs.dtu.dk/ services/TargetP/) were used to identify N-terminal mitochondrial targeting sequences [27, 28]. Multalin (npsa-pbil.ibcp.fr/cgi-bin/npsa_automat.pl?page=/NPSA/ npsa_multalin.html) and Genedoc (http://www.nrbsc.org/ gfx/genedoc/index.html) were used to compare the sequences in Figures 1 and 3.

\section{Results}

3.1. LmjF07.440 and LmjF07.430 Are Functional 3-hydroxyacyl-ACP Dehydratases in Yeast. The L. major database (http://www.genedb.org/) contains two sequences with a high degree of similarity to $T$. brucei HTD2 (Q580H9_9TRYP) representing mitochondrial 3-hydroxyacyl-ACP dehydratase (Figure 1). Both LmjF07.0440 and 
LmjF07.0430 are annotated in this database as maoc family dehydratase-like proteins with very high probability values for being identical to TbHTD2 (BlastP E-scores $1.6 \mathrm{e}-30$ and $5.0 \mathrm{e}-29$, resp.). To predict whether the two Leishmania proteins might reside in mitochondria, three separate algorithms were used to analyze their deduced amino acid sequences: MitoProt (MP), PSORT II (PS), and TargetP (TP). The probability (in brackets) that each of the Leishmania proteins would represent a mitochondrial enzyme was calculated and compared to the values obtained for TbHTD2: LmjF07.0440 (MP, 0.9974; PS, 78.3\%; TP, 0.739), LmjF07.0430 (0.7896; 30.4\%; 0.577), and TbHTD2 (0.8086, 43.5\%, 0.699). In addition, both Leishmania proteins also contained the hydratase 2 motif [29] as well as amino acids germane with the active site residues of human mitochondrial dehydratase [30]. Hence, LmjF07.0440 and LmjF07.0430 were promising HTD2 candidates.

To investigate whether LmjF07.0440 and LmjF07.0430 might function as 3-hydroxyacyl-ACP dehydratases, their respective genes were expressed from the promoter of the yeast CTA1 gene for peroxisomal catalase A (Ctalp), which is only slightly derepressed under the glycerol medium conditions used here $[31,32]$. Due to the lowered PS values for LmjF07.0430 compartmentalisation (compared to LmjF07.0440 and TbHTD2), a fungal MLS was fused to the two Leishmania proteins. Yeast $h t d 2 \Delta$ mutant cells were transformed with plasmids for expressing native $\mathrm{Htd} 2 \mathrm{p}$ (positive control), Ctalp (negative control), or the mitochondrially targeted Leishmania proteins LmjF07.0440 and LmjF07.0430. Transformants were grown on SD-Ura medium, and after successively diluting the yeast cells by a factor of ten, a volume of $2.5 \mu \mathrm{L}$ of the dilutions was applied to SD-Ura or SCglycerol media, and the plates were kept at $30^{\circ} \mathrm{C}$ until single colonies were discernible. The results in Figure 2 revealed that mutant cells expressing LmjF07.0440 or LmjF07.0430 were proficient for growth on glycerol, much like the situation with the self-complemented strain, whereas those mutant cells producing excess Ctalp could not grow or divide on glycerol. Hence, LmjF07.0440 and LmjF07.0430 were adequate at substituting for $\mathrm{Htd} 2 \mathrm{p}$ within the $h t d 2 \Delta$ mutant strain and are referred to hereafter as HTD2-1 and HTD2-2, respectively. Unlike T. brucei, which contains only a single 3-hydroxyacyl-ACP dehydratase HTD2, the related species $L$. major contains two functional dehydratases, and this finding is briefly discussed.

3.2. Choosing LmjF27.2440 and LmjF24.2030 as Candidates for Mitochondrial KAR1. The present study is additionally concerned with identifying Leishmania KAR enzymes with physiological 3-oxoacyl-ACP reductase activities. Three candidate $T$. brucei KAR proteins have been described previously [7], including TbKAR1; however, their physiological function has hitherto not been chronicled. A blast search of the L. major database for proteins with similarity to TbKAR1 revealed the existence of several candidates with a significant probability of representing mitochondrial KAR1. The two top scorers, LmjF27.2440 (BlastP E-score 1.4e-73) and LmjF24.2030 (2.1e-05), are
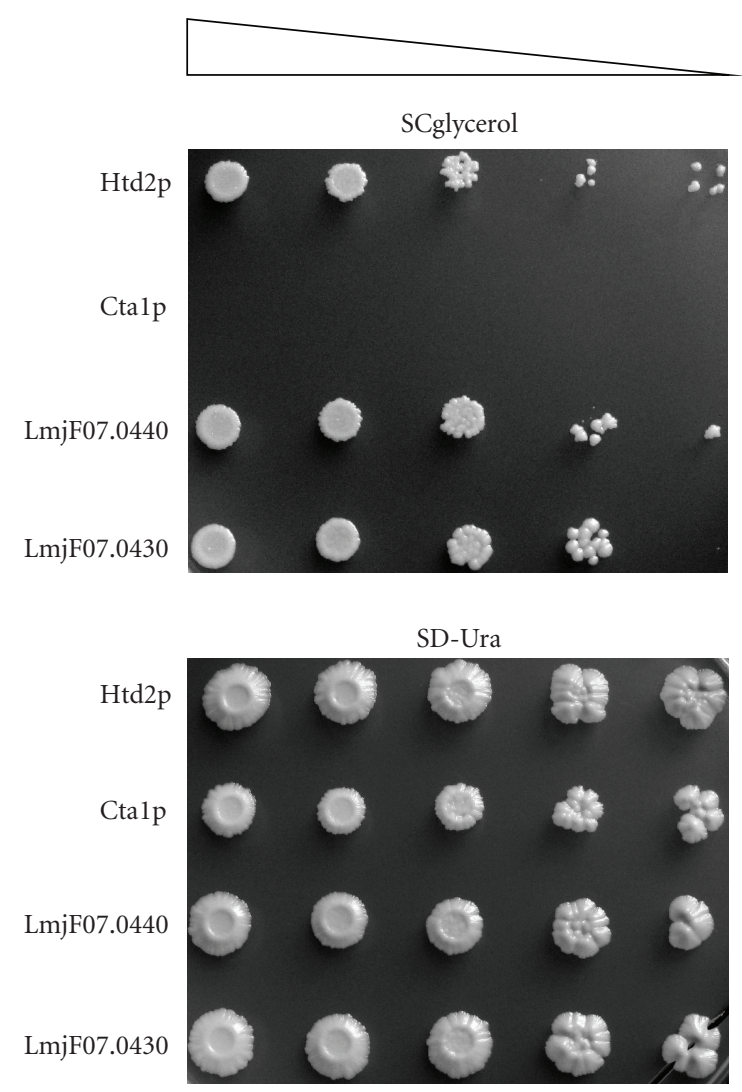

Figure 2: Phenotype rescue of a yeast $h t d 2 \Delta$ strain expressing LmjF07.0440 or LmjF07.0430. Mutant S. cerevisiae BY4741htd2A cells producing native mitochondrial $\mathrm{Htd} 2 \mathrm{p}$, peroxisomal catalase A Ctalp, or each of the mitochondrially targeted Leishmania proteins LmjF07.0440 and LmjF07.0430 were grown on SD-Ura medium selecting for plasmid presence, and following tenfold serial dilution (triangle), the cells were applied to the indicated solid media.

already annotated in the database as putative 3-oxoacylACP reductases. As expected, both proteins were found to be similar to their T. brucei counterpart (Figure 3) and to contain amino acid residues matching those in the catalytic triad of bacterial FabG [33]. The probability that each of the Leishmania proteins would represent a mitochondrial enzyme was similarly estimated on the basis of the algorithms used in the previous section, and the values were compared to those obtained for TbKAR1: LmjF27.2440 (MP, 0.0909; PS, 11.1\%; TP, 0.133), LmjF24.2030 (0.7445; 21.7\%; $0.640)$, and TbKAR1 $(0.8434 ; 13.0 \% ; 0.732)$. These data led to the assessment that LmjF27.2440 and LmjF24.2030 were potential KAR candidates, albeit the former protein, LmjF27.2440, did not appear to be preceded by a recognisable MLS.

3.3. Expression of LmjF27.2440 Rescues the Respiratory Phenotype of oar1 $\triangle$ Cells. To disclose the putative functions of LmjF27.2440 and LmjF24.2030 as 3-oxoacyl-ACP reductases, the corresponding genes were similarly ligated behind the CTA1 promoter. The low prediction values for LmjF27.2440 being a mitochondrial protein meant that a fungal MLS 


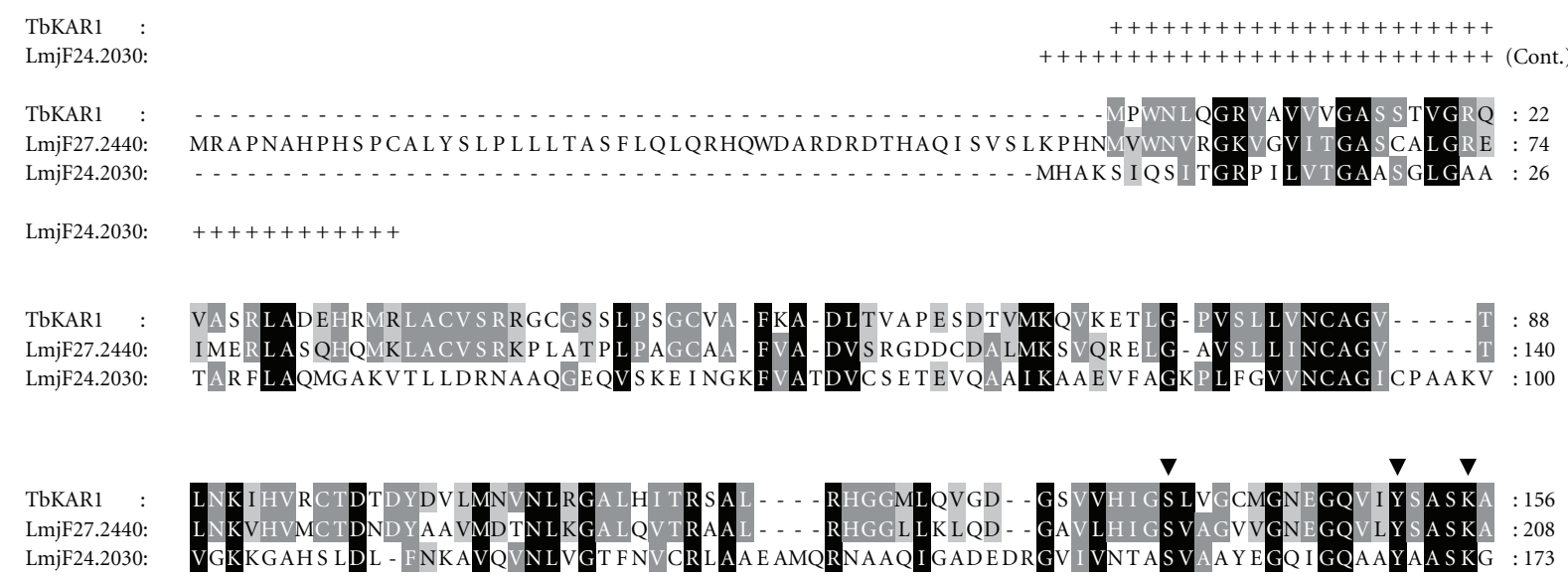

LmjF27.2440:

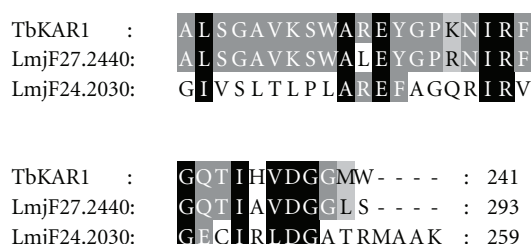

FIGURE 3: Similarity of L. major LmjF24.2440 and LmjF24.2030 to their T. brucei homologue, TbKAR1. MitoProt predictions of cleaved mitochondrial leader sequences are indicated $(+)$. Catalytic amino acid residues occurring in E. coli FabG are marked $(\boldsymbol{\nabla})$. The accession number for TbKAR1 was AAX79999.

was appended to the N-terminus of each of the Leishmania candidates. In the present case, yeast oar1 $\Delta$ mutant cells harbouring plasmids for expressing fungal Oarlp, Ctalp, or mitochondrially targeted Leishmania LmjF27.2440 and LmjF24.2030 were streaked on SCglycerol medium, and this revealed that mutants expressing LmjF27.2440 or Oar1p could grow on glycerol but not those with plasmids for LmjF24.2030 or Ctalp (data not shown).

In a similar strategy to that used in the previous section, the sufficiency of Leishmania LmjF27.2440 at replacing yeast Oarlp was demonstrated using serial dilutions that were applied to SD-Ura or SCglycerol media. The results in Figure 4 revealed that mutant cells expressing LmjF27.2440 were competent for growth on glycerol, to about one tenth of the situation with the self-complemented strain, whereas those mutant cells producing excess Ctalp could not grow or divide on glycerol. Hence, the respiratory growth phenotype of the oar $1 \Delta$ mutant could be rescued, at least in part, by a mitochondrially targeted LmjF27.2440.

\subsection{The Electron Transfer Chain in oar1s Mitochondria is} Restored by LmjF27.2440. S. cerevisiae cells devoid of Oar1p lack assembled cytochrome complexes [13], and so their return to respiratory growth was presumed to coincide with restored cytochrome complexes and a rehabilitated electron transport chain. To demonstrate that the electron transfer chain of mutant cells expressing LmjF27.2440 had been regenerated, respiration competence was monitored with an overlay consisting of 2,3,5-triphenyltetrazolium chloride (TTC) that was applied to the aforementioned SD-Ura plate.
The results revealed that only mutant oar $1 \Delta$ cells expressing Oar1p or LmjF27.2440 could metabolise TTC to generate the red colour, whereas high concentrations of oar $1 \Delta$ cells expressing Ctalp remained white (lower panel; Figure 4). This indicated that within the LmjF27.2440-expressing mutant, at least a partial recovery of the mitochondrial electron transfer chain had been achieved.

3.5. Expression of LmjF27.2440 Resumes Lipoic Acid Production in the oar $1 \Delta$ Mutant. As mentioned in the introduction, trypanosome FASII is involved in the synthesis of the octanoate precursor of lipoic acid [7]. Hence, to couple the expression of LmjF27.2440 with lipoic acid biosynthesis in yeast mitochondria, a biological assay relying on mutant bacterial cells was employed to measure the levels of lipoic acid in the previous three strains (each grown in triplicates). The results showed that extracts produced from the negative control yeast strain (oar1 $\Delta$ cells expressing Cta1p) gave rise to a growth level of these lipoic-acid deficient bacterial cells that was equivalent to $23.3 \pm 2.8 \mathrm{ng}$ lipoic acid per gram wet weight yeast cells (values are mean \pm S.D., $n=3$ ), whereas the lipoic acid present in mutant yeast cells expressing fungal Oar1p or Leishmania LmjF27.2440 promoted bacterial growth to levels corresponding to $294.3 \pm 54.5$ and $59.0 \pm$ $15.4 \mathrm{ng}$ lipoic acid per gram wet weight, respectively. The combined results for LmjF27.2440, referred to hereafter as $L m K A R 1$, were commensurate with the functions of a FASIIlike 3-oxoacyl-ACP reductase, and the implications of this finding are discussed. 

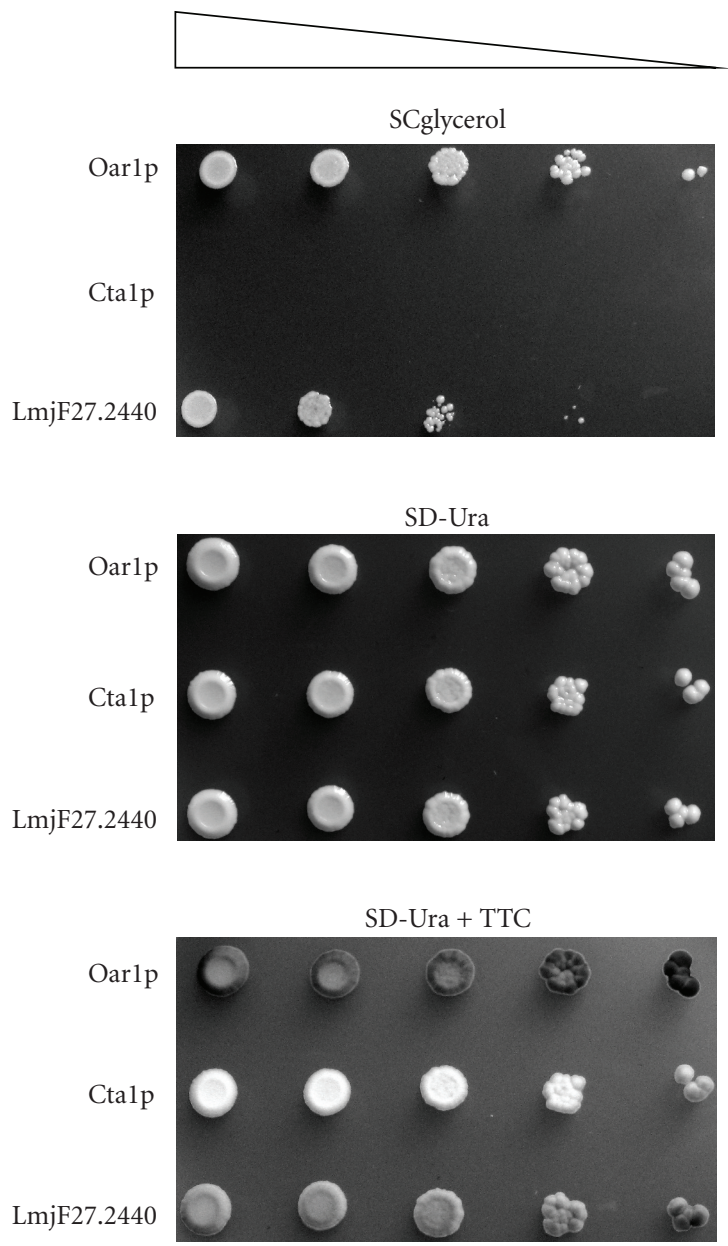

Figure 4: Phenotype rescue of a yeast oarl $\Delta$ strain expressing LmjF27.2440. S. cerevisiae BY4741oarls mutants producing native mitochondrial Oarlp, Cta1p, or mitochondrially targeted LmjF27.2440 were propagated on SD-Ura medium that applied selective pressure for plasmid maintenance, and following sequential tenfold dilution (triangle), the cells were spotted onto the indicated solid media. Respiration competence was examined by applying 2,3,5-triphenyltetrazolium chloride (TTC) as an overlay to the SD-Ura medium.

\section{Discussion}

Although several components of T. brucei FASII have been revealed before, Trypanosoma and Leishmania are sufficiently different to warrant a study dedicated to identifying FASII also in L. major. In the present work, two novel L. major proteins, LmjF07.0440 (HTD2-1) and LmjF07.0430 (HTD22 ), were shown to rescue the mutant phenotype of $S$. cerevisiae $h t d 2 \Delta$ cells with respect to respiratory growth, thereby acting as physiologically functional 3-hydroxacylACP dehydratases. The use of yeast $h t d 2 \Delta$ cells for identifying Trypanosomatidae HTD2 has been established previously [9], affirming that functional complementation studies entail a major experimental advantage over more conventional methods for characterising enzymatic activities. In the aforementioned case of TbHTD2, difficulties in measuring enzyme activities could only be overcome by using a bacterially expressed fusion protein [9]. In order to obviate the lengthy and laborious task of generating soluble Leishmania proteins, complementation of the fungal HTD2 gene was used here instead, and this method provided a very effective alternative for demonstrating the mitochondrial dehydratase function of $L m H T D 2-1$ and $L m H T D 2-2$. Unlike the situation with T. brucei, the observation that L. major contains two HTD2 genes is surprising. However, since there are no data presently available in the Leishmania databases regarding the potential expression of the two genes at different life-cycle stages, the selective advantage behind this gene duplication remains enigmatic.

A further important result to emerge here was the identification of LmjF27.2440 as a physiologically functional 3-oxoacyl-ACP reductase LmKAR1, potentially of mitochondrial FASII. In T. brucei, three different proteins have been chronicled as representing this enzyme, and although they were all experimentally shown to be directed to the parasite's mitochondria [7], no data were presented for their enzyme activities or physiological functions. Here, the ability of LmjF27.2440 to replace Oarlp in oar1 $\Delta$ mutant cells was determined, albeit the extent of the phenotype rescue achieved by ectopically producing LmjF27.2440 was limited. Judging from the difference in respiratory growth of the LmKAR1-rescued mutants compared with the selfcomplemented strain (upper panel; Figure 4), it can be surmised that the former strain grew to a level that was approximately five- to tenfold less than the latter. The reduced respiratory growth seen here was additionally mirrored by a comparatively lower efficiency at metabolising TTC (lower panel; Figure 4). Moreover, a fivefold difference in levels of lipoic acid production could also be discerned between the two strains. The reason for this apparent discrepancy remains unclear, since by using an identical approach with the $h t d 2 \Delta$ mutant, its phenotype could be rescued by $L m H T D 2-1$ or $L m H T D 2-2$ essentially to the level achieved by native $\mathrm{Htd} 2 \mathrm{p}$. One possible explanation leans on the efficacy of mitochondrial targeting.

The poor predictability regarding the potential mitochondrial targeting of LmjF27.2440 prompted the addition of a fungal MLS to the N-terminus of the protein. In this respect it should be noted that although the process of importing proteins into the mitochondria is well conserved across eukaryotes, some differences do exist, especially between yeast and mammals [34]. Since an exhaustive discourse on these differences is beyond the scope of this discussion, it can at least be speculated that in the situation of a lack of a formal MLS, LmjF27.2440 might actually contain a cryptic signal for compartmentalisation (beginning with the amino acid residue at position 53; Figure 3), which may or may not be recognised by yeast. The potentially deleterious effect of adding a fungal MLS on top of such a cryptic signal cannot be presently assessed. While the ultimate confirmation for mitochondrial localisation of LmKAR1, but also LmHTD2-1 and LmHTD2-2, will require dedicated studies on Leishmania using specific antibodies or GFP fusion proteins, within the context of yeast it is not 
uncommon to submit to the temptation of ensuring that heterologous mitochondrial proteins be correctly targeted by artificially appending an additional fungal MLS [14]. Nevertheless, when considering the situation in T. brucei, whereby the KAR1 [7] and HTD2 [9] proteins have been convincingly localised to the mitochondria, it is tempting to speculate that the analogous L. major components will also be similarly compartmentalised.

In reference to the number of KAR enzymes present in FASII of Leishmania, the second protein tested, LmjF24.2030, which was predicted to be a mitochondrial protein, nevertheless, failed to rescue the mutant phenotype. In the context of a yeast system, this observation alone is not sufficient to discount completely the possibility that LmjF24.2030 might still represent a functional KAR in Leishmania. However, it was no longer attractive to pursue LmjF24.2030 any further once it failed to rescue the yeast mutant phenotype, since it was conspicuously less similar to TbKAR1 compared with LmjF27.2440 (Figure 3). To conclude, the present identification of three probable FASII components in $L$. major will enrich our knowledge of the molecular biology of an organism threatening the health of hundreds of millions of people worldwide and may help expose its mitochondrial FASII system to novel drug design.

\section{Acknowledgments}

Daniel Paape and Toni Aebischer (both from the University of Edinburgh, UK) are thanked for providing L. major genomic DNA. The author is grateful to J. Kalervo Hiltunen for making this study possible and for providing assistance whenever required and to Alexander J. Kastaniotis and Katri Näppä for valuable advice and the lipoic acid measurements (all from the University of Oulu, Finland). This work was supported by Grant no. P20764-B03 from the Austrian Science Fund (FWF).

\section{References}

[1] B. L. Herwaldt, "Leishmaniasis," The Lancet, vol. 354, no. 9185, pp. 1191-1199, 1999.

[2] H. W. Murray, J. D. Berman, C. R. Davies, and N. G. Saravia, "Advances in leishmaniasis," The Lancet, vol. 366, no. 9496, pp. 1561-1577, 2005.

[3] J. W. Campbell and J. E. Cronan Jr., "Bacterial fatty acid biosynthesis: targets for antibacterial drug discovery," Annual Review of Microbiology, vol. 55, pp. 305-332, 2001.

[4] R. J. Heath, S. W. White, and C. O. Rock, "Inhibitors of fatty acid synthesis as antimicrobial chemotherapeutics," Applied Microbiology and Biotechnology, vol. 58, no. 6, pp. 695-703, 2002.

[5] K. S. Paul, C. J. Bacchi, and P. T. Englund, "Multiple triclosan targets in Trypanosoma brucei," Eukaryotic Cell, vol. 3, no. 4, pp. 855-861, 2004.

[6] A. C. Ivens, C. S. Peacock, E. A. Worthey, et al., "The genome of the kinetoplastid parasite, Leishmania major," Science, vol. 309, no. 5733, pp. 436-442, 2005.

[7] J. L. Stephens, S. H. Lee, K. S. Paul, and P. T. Englund, "Mitochondrial fatty acid synthesis in Trypanosoma brucei,"
Journal of Biological Chemistry, vol. 282, no. 7, pp. 4427-4436, 2007.

[8] J. L. Guler, E. Kriegova, T. K. Smith, J. Lukeš, and P. T. Englund, "Mitochondrial fatty acid synthesis is required for normal mitochondrial morphology and function in Trypanosoma brucei," Molecular Microbiology, vol. 67, no. 5, pp. 1125-1142, 2008.

[9] K. J. Autio, J. L. Guler, A. J. Kastaniotis, P. T. Englund, and J. K. Hiltunen, "The 3-hydroxyacyl-ACP dehydratase of mitochondrial fatty acid synthesis in Trypanosoma brucei," FEBS Letters, vol. 582, no. 5, pp. 729-733, 2008.

[10] J. K. Hiltunen, F. Okubo, V. A. S. Kursu, K. J. Autio, and A. J. Kastaniotis, "Mitochondrial fatty acid synthesis and maintenance of respiratory competent mitochondria in yeast," Biochemical Society Transactions, vol. 33, no. 5, pp. 1162-1165, 2005.

[11] J. K. Hiltunen, M. S. Schonauer, K. J. Autio, T. M. Mittelmeier, A. J. Kastaniotis, and C. L. Dieckmann, "Mitochondrial fatty acid synthesis type II: more than just fatty acids," Journal of Biological Chemistry, vol. 284, no. 14, pp. 9011-9015, 2009.

[12] A. J. Kastaniotis, K. J. Autio, R. T. Sormunen, and J. K. Hiltunen, "Htd2p/Yhr067p is a yeast 3-hydroxyacyl-ACP dehydratase essential for mitochondrial function and morphology," Molecular Microbiology, vol. 53, no. 5, pp. 14071421, 2004.

[13] R. Schneider, B. Brors, F. Burger, S. Camrath, and H. Weiss, "Two genes of the putative mitochondrial fatty acid synthase in the genome of Saccharomyces cerevisiae," Current Genetics, vol. 32, no. 6, pp. 384-388, 1997.

[14] Z. Chen, A. J. Kastaniotis, I. J. Miinalainen, V. Rajaram, R. K. Wierenga, and J. K. Hiltunen, " $17 \beta$-hydroxysteroid dehydrogenase type 8 and carbonyl reductase type 4 assemble as a ketoacyl reductase of human mitochondrial FAS," FASEB Journal, vol. 23, no. 11, pp. 3682-3691, 2009.

[15] A. Gurvitz, "Caenorhabditis elegans F09E10.3 encodes a putative 3-oxoacyl-thioester reductase of mitochondrial type 2 fatty acid synthase FASII that is functional in yeast," Journal of Biomedicine and Biotechnology, vol. 2009, Article ID 235868, 6 pages, 2009.

[16] D.-C. Chen, B.-C. Yang, and T.-T. Kuo, "One-step transformation of yeast in stationary phase," Current Genetics, vol. 21, no. 1, pp. 83-84, 1992.

[17] J. E. Hill, A. M. Myers, T. J. Koerner, and A. Tzagoloff, "Yeast/E. coli shuttle vectors with multiple unique restriction sites," Yeast, vol. 2, no. 3, pp. 163-167, 1986.

[18] S. A. Flippula, R. T. Sormunen, A. Hartig, W.-H. Kunau, and J. K. Hiltunen, "Changing stereochemistry for a metabolic pathway in vivo. Experiments with the peroxisomal $\beta$-oxidation in yeast," Journal of Biological Chemistry, vol. 270, no. 46, pp. 27453-27457, 1995.

[19] F. M. Ausubel, R. Brent, R. E. Kingston, et al., Current Protocols in Molecular Biology, John Wiley \& Sons, New York, NY, USA, 1989.

[20] A. Gurvitz, J. K. Hiltunen, and A. J. Kastaniotis, "Function of heterologous Mycobacterium tuberculosis InhA, a type 2 fatty acid synthase enzyme involved in extending $\mathrm{C}_{20}$ fatty acids to $\mathrm{C}_{60}$-to- $\mathrm{C}_{90}$ mycolic acids, during de novo lipoic acid synthesis in Saccharomyces cerevisiae," Applied and Environmental Microbiology, vol. 74, no. 16, pp. 5078-5085, 2008.

[21] A. Y. Hsu, W. W. Poon, J. A. Shepherd, D. C. Myles, and C. F. Clarke, "Complementation of coq3 mutant yeast by mitochondrial targeting of the Escherichia coli UbiG polypeptide: evidence that UbiG catalyzes both $O$-methylation steps in 
ubiquinone biosynthesis," Biochemistry, vol. 35, no. 30, pp. 9797-9806, 1996.

[22] M. D. Rose, F. Winston, and P. Heiter, Methods in Yeast Genetics: A Laboratory Manual, Cold Spring Harbor Laboratory Press, Cold Spring Harbor, NY, USA, 1990.

[23] J. Sambrook, E. F. Fritsch, and T. Maniatis, Molecular Cloning: A Laboratory Manual, Cold Spring Harbor Laboratory Press, Cold Spring Harbor, NY, USA, 1989.

[24] E. Böker-Schmitt, S. Francisci, and R. J. Schweyen, "Mutations releasing mitochondrial biogenesis from glucose repression in Saccharomyces cerevisiae," Journal of Bacteriology, vol. 151, no. 1, pp. 303-310, 1982.

[25] M. A. Hayden, I. Y. Huang, G. Iliopoulos, M. Orozco, and G. W. Ashley, "Biosynthesis of lipoic acid: characterization of the lipoic acid auxotrophs Escherichia coli W1485-lip2 and JRG33lip9," Biochemistry, vol. 32, no. 14, pp. 3778-3782, 1993.

[26] S. Brody, C. Oh, U. Hoja, and E. Schweizer, "Mitochondrial acyl carrier protein is involved in lipoic acid synthesis in Saccharomyces cerevisiae," FEBS Letters, vol. 408, no. 2, pp. 217-220, 1997.

[27] M. G. Claros and P. Vincens, "Computational method to predict mitochondrially imported proteins and their targeting sequences," European Journal of Biochemistry, vol. 241, no. 3, pp. 779-786, 1996.

[28] O. Emanuelsson, H. Nielsen, S. Brunak, and G. von Heijne, "Predicting subcellular localization of proteins based on their N-terminal amino acid sequence," Journal of Molecular Biology, vol. 300, no. 4, pp. 1005-1016, 2000.

[29] Y.-M. Qin, A. M. Haapalainen, S. H. Kilpeläinen, et al., "Human peroxisomal multifunctional enzyme type 2. Sitedirected mutagenesis studies show the importance of two protic residues for 2-enoyl- CoA hydratase 2 activity," Journal of Biological Chemistry, vol. 275, no. 7, pp. 4965-4972, 2000.

[30] K. J. Autio, A. J. Kastaniotis, H. Pospiech, et al., "An ancient genetic link between vertebrate mitochondrial fatty acid synthesis and RNA processing," FASEB Journal, vol. 22, no. 2, pp. 569-578, 2008.

[31] M. Filipits, M. M. Simon, W. Rapatz, B. Hamilton, and H. Ruis, "A Saccharomyces cerevisiae upstream activating sequence mediates induction of peroxisome proliferation by fatty acids," Gene, vol. 132, no. 1, pp. 49-55, 1993.

[32] H. Rottensteiner, A. J. Kal, M. Filipits, et al., "Pip2p: a transcriptional regulator of peroxisome proliferation in the yeast Saccharomyces cerevisiae," EMBO Journal, vol. 15, no. 12, pp. 2924-2934, 1996.

[33] A. C. Price, Y.-M. Zhang, C. O. Rock, and S. W. White, "Structure of $\beta$-ketoacyl-[acyl carrier protein] reductase from Escherichia coli: negative cooperativity and its structural basis," Biochemistry, vol. 40, no. 43, pp. 12772-12781, 2001.

[34] M. Mori and K. Terada, "Mitochondrial protein import in animals," Biochimica et Biophysica Acta, vol. 1403, no. 1, pp. 12-27, 1998. 

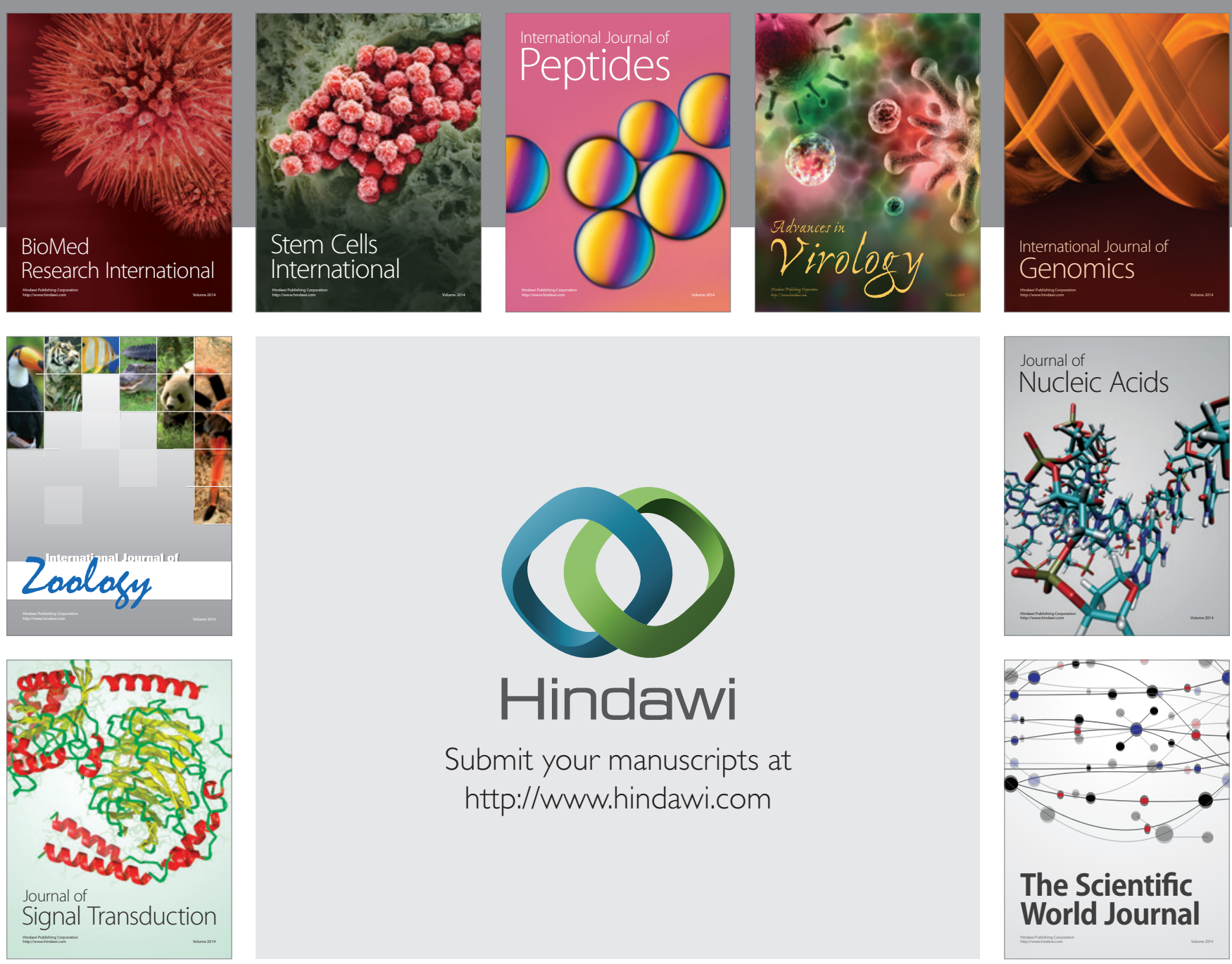

Submit your manuscripts at

http://www.hindawi.com
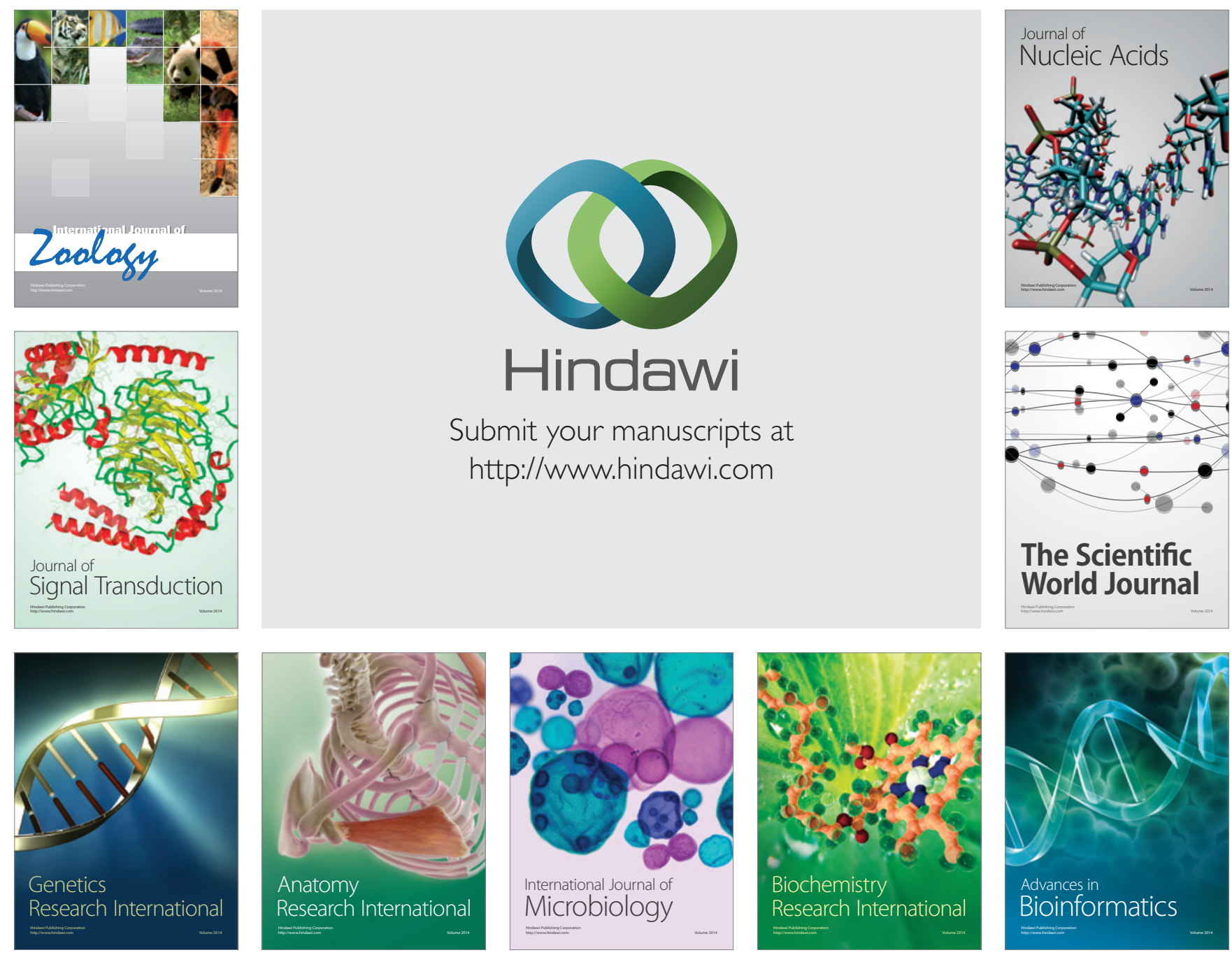

The Scientific World Journal
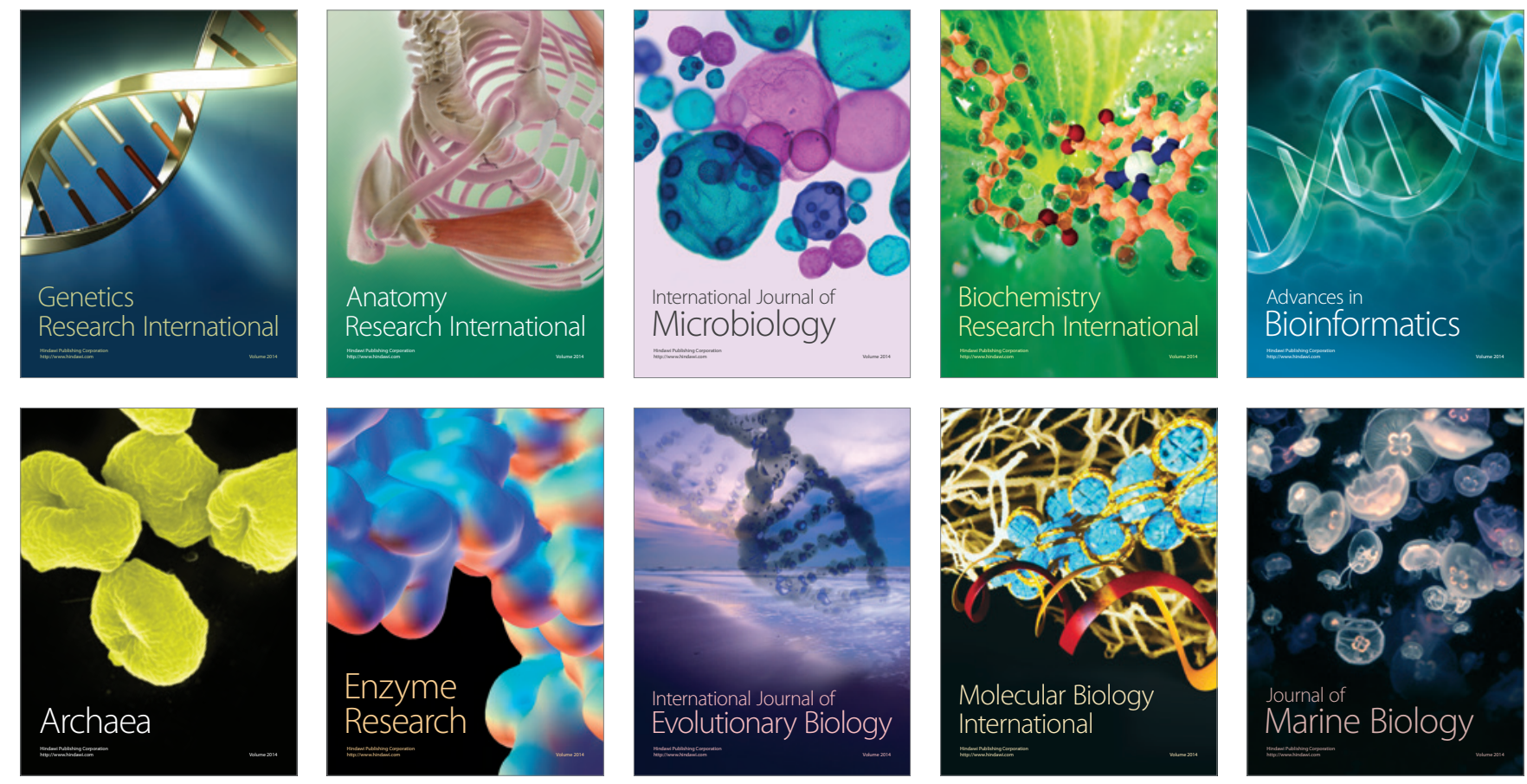